\title{
Osteoporoseprophylaxe mit Kalzium und Vitamin D nutzlos
}

Die Einnahme von Kalzium- und/oder Vitamin-D-Präparaten führt einer Metaanalyse zufolge nicht zu einer Abnahme des Frakturrisikos im Alter.

Obwohl ein Mangel an Kalzium und Vitamin D zu den Hauptursachen einer primären Osteoporose gezählt wird, scheint sich eine routinemäßige Supplementation für ältere Menschen nicht in einem geringeren Frakturrisiko niederzuschlagen. Selbst Menschen mit Risikofaktoren für eine Osteoporose haben gemäß einer in JAMA veröffentlichten Metaanalyse (1) keinen Nutzen von der Einnahme entsprechender Präparate.

In die Analyse wurden ausschließlich randomisierte kontrollierte Studien einbezogen, insgesamt 33 mit zusammen 51.145 Teilnehmern. Die Studienteilnehmer waren über 50 Jahre alt und lebten außerhalb von Heimen. Nur an wenigen Studien waren explizit Patienten mit Osteoporose beteiligt, in einige waren Personen mit Risikofaktoren aufgenommen worden, etwa mit niedrigem 25-Hydroxy-Vitamin-D3-Spiegel oder geringer Kalziumzufuhr mit der Nahrung. Der Effekt der Supplementierung wurde an dem einer Placebo- oder Nichtsupplementierung gemessen. Patienten mit kortikosteroidinduzierter Osteoporose sowie Patienten, die zusätzlich Osteoporosemedikamente erhielten, waren ausgeschlossen.

\section{Kein Einfluss auf Risiko von \\ Hüftfrakturen}

Wegen der besonders gravierenden Folgen wurden Hüftfrakturen als primärer Studienendpunkt gewählt. Das Auftreten dieses Endpunktes wurde weder durch Kalzium- noch durch Vitamin-D- oder Kombinationspräparate signifikant reduziert. Tatsächlich lag das Risiko vor allem bei alleiniger Kalzium- ergänzung numerisch sogar höher (um $53 \%)$, der Unterschied war jedoch statistisch nicht bedeutsam. Bei der Auswertung von vertebralen, nicht-vertebralen oder Frakturen insgesamt waren ebenfalls keine Vorteile einer Supplementierung erkennbar. Auch ein Ausschluss von Studien geringerer Qualität änderte nichts an diesem Ergebnis.

\section{Auch in Patientensubgruppen kein Nutzen}

Ein positiver Effekt blieb selbst dann aus, wenn Subgruppen von Studienteilnehmern betrachtet wurden. Für das Frakturrisiko spielte es keine Rolle, in welchen Dosierungen die Präparate eingesetzt wurden (Kalzium $\geq$ oder $<1 \mathrm{~g} /$ Tag, Vitamin D $\geq$ oder $<800$ IE/Tag), ob die Patienten früher bereits Frakturen erlitten hatten oder wie hoch die Kalziumzufuhr mit der Nahrung ( $\geq$ oder $<900 \mathrm{mg} / \mathrm{Tag}$ ) oder der anfängliche 25-OH-Vitamin-D3-Spiegel ( $\geq$ oder $<20 \mathrm{ng} / \mathrm{ml}$ ) waren. Eine Ausnahme bildeten Patienten mit einem 25-OH-Vitamin-D3-Spiegel $\geq 20 \mathrm{ng} / \mathrm{ml}$ : Bei ihnen war eine Vitamin-D-Ergänzung sogar mit einer um $49 \%$ erhöhten Rate von Hüftfrakturen assoziiert.

》) Selbst Menschen mit Osteoporoserisiko profitieren nicht von der Einnahme entsprechender Präparate

„Diese Ergebnisse sprechen nicht für einen routinemäßigen Gebrauch von Kalzium- und Vitamin-D-Supplementen bei älteren, nicht in Heimen lebenden Menschen", lautet das Fazit der Studienautoren. Ein Manko der Analyse ist-neben den üblichen Problemen, die die gemeinsame Auswertung von heterogenen Studien mit sich bringt unter anderem die unvollständige Erfassung der anfänglichen 25-OH-Vitamin-D3-Spiegel.

Das Fazit widerspricht der DVO (Dachverband Osteologie)-Leitlinie von 2017. Danach sollte zur Osteoporoseprophylaxe die Zufuhr einer ausreichenden Menge an Kalzium und Vitamin D sichergestellt werden. Wenn die empfohlene Zufuhr von 1000 mg Kalzium und 800 IE Vitamin D pro Tag mit der Ernährung nicht sicher erreicht werden kann, werden Supplemente empfohlen. Von der isolierten Zufuhr von Vitamin D wird allerdings abgeraten.

\section{Literatur \\ 1. Zhao JG et al (2017) Association Between Calcium or Vitamin D Supplementation and Fracture Incidence in Community-Dwelling Older Adults: A Systematic Review and Meta-analysis. JAMA 318(24):2466-2482}

rheuma plus $2018 \cdot 17: 149$

https://doi.org/10.1007/s12688-0180204-0

(c) Springer-Verlag GmbH Austria, ein Teil von Springer Nature 2018 